\title{
Single-Centre Results of Treatment of Retroperitoneal and Mesenteric Cystic Lymphangiomas
}

\author{
Chih-Ming Su ${ }^{a}$ Ming-Chin Yu ${ }^{a, c}$ Huang-Yang Chen ${ }^{d}$ Jeng-Hwei Tseng ${ }^{b}$ \\ Yi-Yin Jan ${ }^{\mathrm{a}}$ Miin-Fu Chen ${ }^{\mathrm{a}}$ \\ Departments of a Surgery and ${ }^{\mathrm{b}}$ Radiology, Chang Gung Memorial Hospital, Chang Gung University, Taipei, \\ 'Graduate Institute of Clinical Medical Sciences, Chang Gung University, Taipei, and d Department of Surgery, \\ Chang Gung Memorial Hospital, Keelung, Taiwan, ROC
}

\section{Key Words}

Intra-abdominal cystic lymphangiomas $\cdot$ Mesenteric cystic lymphangioma $\cdot$ Retroperitoneal cystic lymphoma

\begin{abstract}
Background: Intra-abdominal cystic lymphangiomas are rare and usually present as benign large cystic masses. The treatment of choice of mesenteric and retroperitoneal cystic lymphangiomas is surgical resection. Methods: Seventeen adults, 11 male and 6 female, with a median age of 39 years were investigated. Presentation, treatment, and outcomes of the mesenteric and retroperitoneal cystic lymphangiomas were analyzed. Results: The most common symptom was abdominal pain. The median tumour size was $12.0 \mathrm{~cm}$ in diameter. Patients with retroperitoneal lymphangiomas were younger $(p=0.043)$. However, 4 out of 8 patients with the mesenteric type required bowel resection $(p=0.064)$. Magnetic resonance imaging allowed a good differentiation of cystic and septal structures. No postoperative complications occurred, but patients with mesenteric types had longer postoperative stays ( 7.0 vs. 13.5 days; $p<0.001$ ). The long-term outcome of both groups was satisfactory. Conclusions: The surgical results were good with symptom relief.
\end{abstract}

Bowel resection was common in mesenteric cystic lymphangiomas. Because of its higher resolution, magnetic resonance imaging is suggested.

Copyright $\odot 2007$ S. Karger AG, Basel

\section{Introduction}

Cystic lymphangiomas, mostly occurring in children, are rare and are lymphatic developmental anomalies. Cystic hygromas located in the neck area are the most common tumours, but other cystic lymphangiomas of axilla, thoracic cavity, and abdomen have been also reported [1]. Intra-abdominal cystic lymphangiomas may arise from the retroperitoneum, the mesentery, and visceral organs. The common presentations of intra-abdominal cystic lymphangiomas are abdominal mass and distension, and most have cystic and septal components [2]. Recent reports have been limited to small series and case reports. Although mesenteric and retroperitoneal cystic lymphangiomas have been reported, the difference between the two types has been seldom investigated. Clinical presentations, surgical treatment, and longterm outcomes of 17 patients representing the two types 
of lymphangiomas are described in this study. The diagnostic tools, including sonography, computed tomography (CT), and magnetic resonance imaging (MRI), are also described. The results of this study showed that the mesenteric type frequently requires bowel resection and that the hospital stay for optimum patient recovery is longer.

\section{Patients and Methods}

From 1985 to 2003, 29 patients underwent surgical resection for lymphangiomas. Seventeen adults, 6 males and 11 females, were included in this study. Retroperitoneal cystic lymphangiomas were defined as tumours of the retroperitoneum or tumours involving retroperitoneal organs. Tumours that only involved the mesentery were defined as mesenteric cystic lymphangiomas. Other tumours involving visceral organs and omentum were rare and were excluded. The pre-operative evaluation of the lymphangiomas included abdominal ultrasonography, CT, and MRI. Patients who had undergone surgical resection were enrolled. No patients succumbed within 1 month postoperatively, and no perioperative morbidity occurred. Demographic data and the clinical picture were reviewed.

The final diagnosis was confirmed by the typical pathological findings: flat epithelial endothelium, cystic wall of lymphoid tissue, small lymphatic space or smooth muscle, and foam cells in the cystic wall. No recurrence was reported in the follow-up image studies. The median follow-up period was 50.0 (range 3-240) months.

SPSS version 12.0 (SPSS, Chicago Ill., USA) was used to analyze the differences between these two groups. The clinicopathological variables, demographic data, clinical presentations, operative treatments, and pathological features were included in the nonparametric statistical analysis. Data were analyzed by MannWhitney U test and chi-square test with Yates' correction. $\mathrm{p}<0.05$ was considered statistically significant.

\section{Results}

Table 1 summarizes the clinical characteristics of the patients. Most patients had insidious onset of abdominal discomfort, and 1 patient had an incidental finding by routine ultrasound examination. Six cases had lower abdominal or pelvic retroperitoneal cystic lymphangiomas, and 4 and 5 patients were diagnosed as having intestinal mesenteric and mesocolonic lymphangiomas, respectively. One patient presented with a lymphangioma in the lymphoid tissue of the coeliac trunk, and 1 patient had a lymphangioma in the peripancreatic area. Two patients had an acute abdomen due to torsion of mesenteric cysts and intestinal obstruction. No patients suffered from fever, body weight loss, or gastro-intestinal bleeding. One patient with a mesenteric cystic lymphangioma had leucocytosis prior to the surgical intervention. The levels of the tumour markers carcino-embryonic antigen, $\alpha$-fetoprotein, and cancer antigen 125 were within the normal ranges. All patients underwent surgery, and the shortterm outcome was good. Visceral organs were preserved, and tumours were safely resected without marsupialization. Postoperative drains showed clear fluid without chylous ascites. The long-term follow-up was satisfactory without recurrence by sonography. However, 1 patient had undergone surgery for ventral hernia, and 1 patient had developed adhesion ileus at follow-up examination. The data shown in table 1 concerning children treated in our institute were taken from the study performed by Luo et al. [3], with permission.

Table 2 depicts the differences between retroperitoneal and mesenteric cystic lymphangiomas. Patients with mesenteric cystic lymphangiomas were older, and 4 out of 8 patients underwent intestinal resection. Two patients in the mesenteric cystic lymphangioma group had intestinal obstruction, and 1 in the retroperitoneal cystic lymphangioma group had ovarian torsion necessitating emergency surgery. The hospital stay required by the mesenteric lymphangioma group was also longer than that of the retroperitoneal lymphangioma group.

Imaging studies revealed that 5 of 11 patients (45.5\%) and 3 of 9 patients (33.3\%) had typical septal and cystic structures by sonography and CT studies, respectively. The septal structure in the mesenteric lymphangioma group did not significantly differ from that of the retroperitoneal lymphangioma group. Moreover, MRI had a superior resolution for depicting the soft-tissue structure. Figures 1-3 show MRI scans of a retroperitoneal lymphangioma. The 25-year-old female had insidious onset of abdominal discomfort. MRI revealed cystic and septal components. The spatial relationship between the mass and visceral organs is clearly shown. The recovery of this patient after surgical resection was good.

\section{Discussion}

Large series of intra-abdominal cystic lymphangiomas are rarely reported in the medical literature. Less than $1 \%$ of the cystic lymphangiomas are found in the retroperitoneum, and the rare lymphangiomas occurring in the mesentery have been classified into six groups by de Perrot et al. [4]. Losanoff et al. [2] defined four different types of mesenteric cystic lymphangiomas, including pedicled, sessile, retroperitoneal extended, and multicen- 
Table 1. Clinical presentation of mesenteric and retroperitoneal cystic lymphangiomas
Table 2. Clinical picture and treatment of the intra-abdominal lymphangiomas

\begin{tabular}{lll}
\hline $\begin{array}{l}\text { Demographic data and } \\
\text { symptoms and signs }\end{array}$ & Adults & Children [3] \\
\hline Median age, years (range) & $39(19-76)$ & $3.55($ from birth to 6) \\
Male/female ratio & $6(35.3 \%) / 11(64.7 \%)$ & $7(58.3 \%) / 5(41.7 \%)$ \\
Mesenteric/retroperitoneum & $8(47.2 \%) / 9(52.8 \%)$ & $5(71.4 \%) / 2(28.6 \%)^{\mathrm{a}}$ \\
\hline Symptoms and signs & & \\
Abdominal pain & $10(58.9 \%)$ & $5(41.7 \%)$ \\
Abdominal distension & $4(23.5 \%)$ & $1(8.3 \%)$ \\
Palpable mass & $6(35.3 \%)$ & $3(25.0 \%)$ \\
Acute abdomen & $2(11.8 \%)^{\mathrm{b}}$ & $1(8.3 \%)^{\mathrm{c}}$ \\
Median size, cm (range) & $12.0(3.0-40.0)$ & $8.5(6.0-20.0)$ \\
\hline
\end{tabular}

${ }^{a}$ Five cases had omentum cystic lymphangiomas.

${ }^{\mathrm{b}}$ One adult with intestinal obstruction had surgery and 1 had emergency operation due to cyst torsion.

${ }^{c}$ One child presenting with a lymphangioma mimicking appendicitis required surgical intervention.

\begin{tabular}{lccr}
\hline $\begin{array}{l}\text { Demographic data } \\
\text { and outcomes }\end{array}$ & Retroperitoneal & Mesenteric & p \\
\hline Median age, years (range) & $29(19-50)$ & $50.5(19-76)$ & 0.043 \\
Male/female ratio & $2 / 7$ & $4 / 4$ & 0.492 \\
Abdominal pain & $6(66.7 \%)$ & $5(62.5 \%)$ & 1.000 \\
Abdominal fullness & $2(22.2 \%)$ & $2(25.0 \%)$ & 1.000 \\
Mass & $3(33.3 \%)$ & $3(37.5 \%)$ & 1.000 \\
\hline Surgery and outcome & & & \\
Median size, cm (range) & $10(3-30)$ & $12.5(8-40)$ & 0.093 \\
Bowel resection & $0(0.0 \%)$ & $4(50.0 \%)$ & 0.064 \\
Median hospital stay, days (range) & $7(4-10)$ & $13.5(9-27)$ & $<0.001$ \\
Median postoperative stay, days (range) & $5(3-7)$ & $8.5(6-13)$ & 0.006 \\
\hline
\end{tabular}

Mann-Whitney U test and chi-square test with Yates' correction were used to analyze the data. tric lymphangiomas [2]. In this series, all cases were of the sessile or extended types, with adhesion to major vessels such as the coeliac trunk. The latter belonged to the retroperitoneal cystic lymphangiomas in this study because of the involvement of major vessels. Luo et al. [3] reported 12 paediatric patients with intra-abdominal cystic lymphangiomas. Most had abdominal pain, abdominal distension, or palpable mass, and some had acute abdomen mimicking appendicitis. Table 1 summarizes the data for 17 cases of intra-abdominal cystic lymphangiomas occurring over an 18-year period and compares the indications between adults and children. The clinical symptoms and signs of the adult patients were similar to those of the paediatric patients. A series of 6 cases dem- onstrated that children usually present with mesenteric cysts and that a higher proportion of adults had an increased risk of retroperitoneal cystic lymphangiomas [5]. However, the case number was limited. According to this study and the paediatric series reported by Luo et al. [3], $52.8 \%$ of the adults and $28.6 \%$ of the children had retroperitoneal cystic lymphangiomas. This is consistent with the series reported by de Perrot et al. [5] where intra-abdominal cystic lymphangiomas in adults usually presented as retroperitoneal lesions.

Retroperitoneal cystic lymphangiomas are seldom reported, but the pathological characteristics are similar to those of the mesenteric type [6, 7]. The reported clinical features, such as abdominal pain, fullness, and disten- 


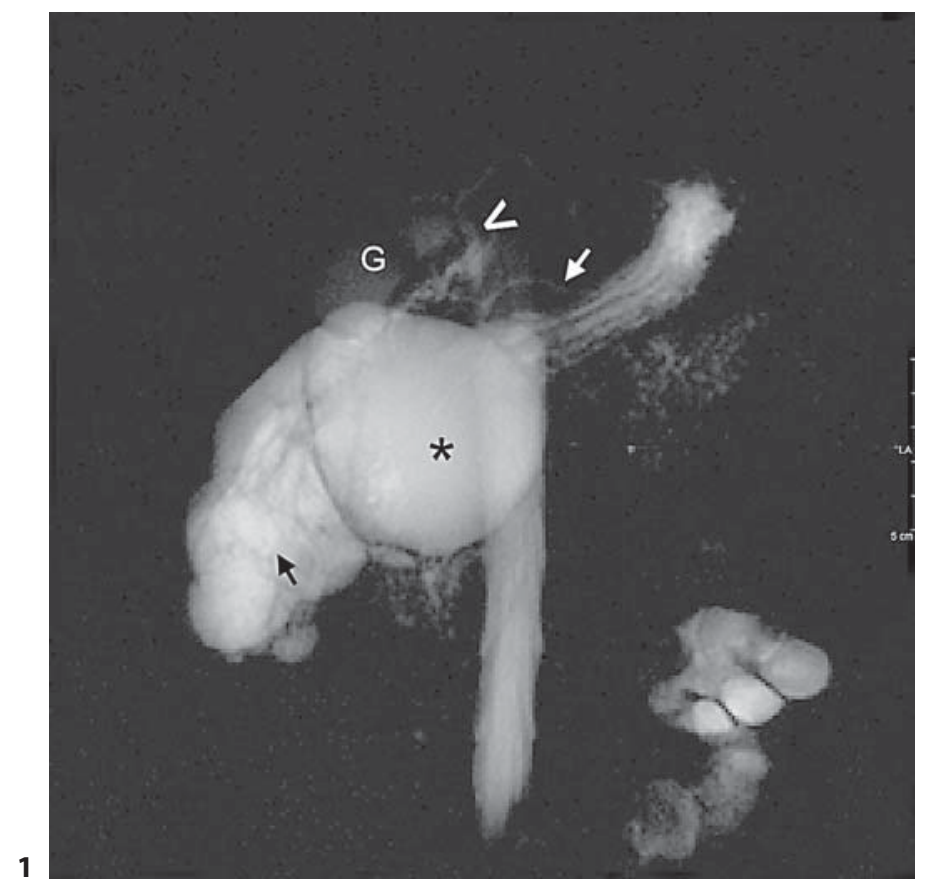

Fig. 1. A 25-year-old female with retroperitoneal lymphangioma. Coronal projectile magnetic resonance cholangiopancreatography shows the gallbladder $(\mathrm{G})$ and a large cystic lymphangioma composed of a large cyst (asterisk) and multiple small cysts within diffuse thin septations (dark arrow). The common bile duct (arrowhead) and the main pancreatic duct (white arrow) are also shown. Fig. 2. Sagittal $\mathrm{T}_{2}$-weighted fast spin echo shows the relationship between the gallbladder (asterisk) and the large cystic lymphangioma with diffuse thin septations (arrow).

Fig. 3. Coronal-plane gadolinium-enhanced T1-weighted spoiled gradient echo shows that the lymphangioma is composed of a large cyst (asterisk) and multiple small cysts with diffuse thin septations (arrow).

sion, are similar to those of the mesenteric lymphangiomas of the patients presented in this study. Patients with retroperitoneal cystic lymphangiomas, commonly female, were younger than those with mesenteric lymphangiomas. Pelvic cystic lymphangiomas were confirmed in 6 patients who routinely underwent gynaecologic sonography. Therefore, the age of onset was younger and
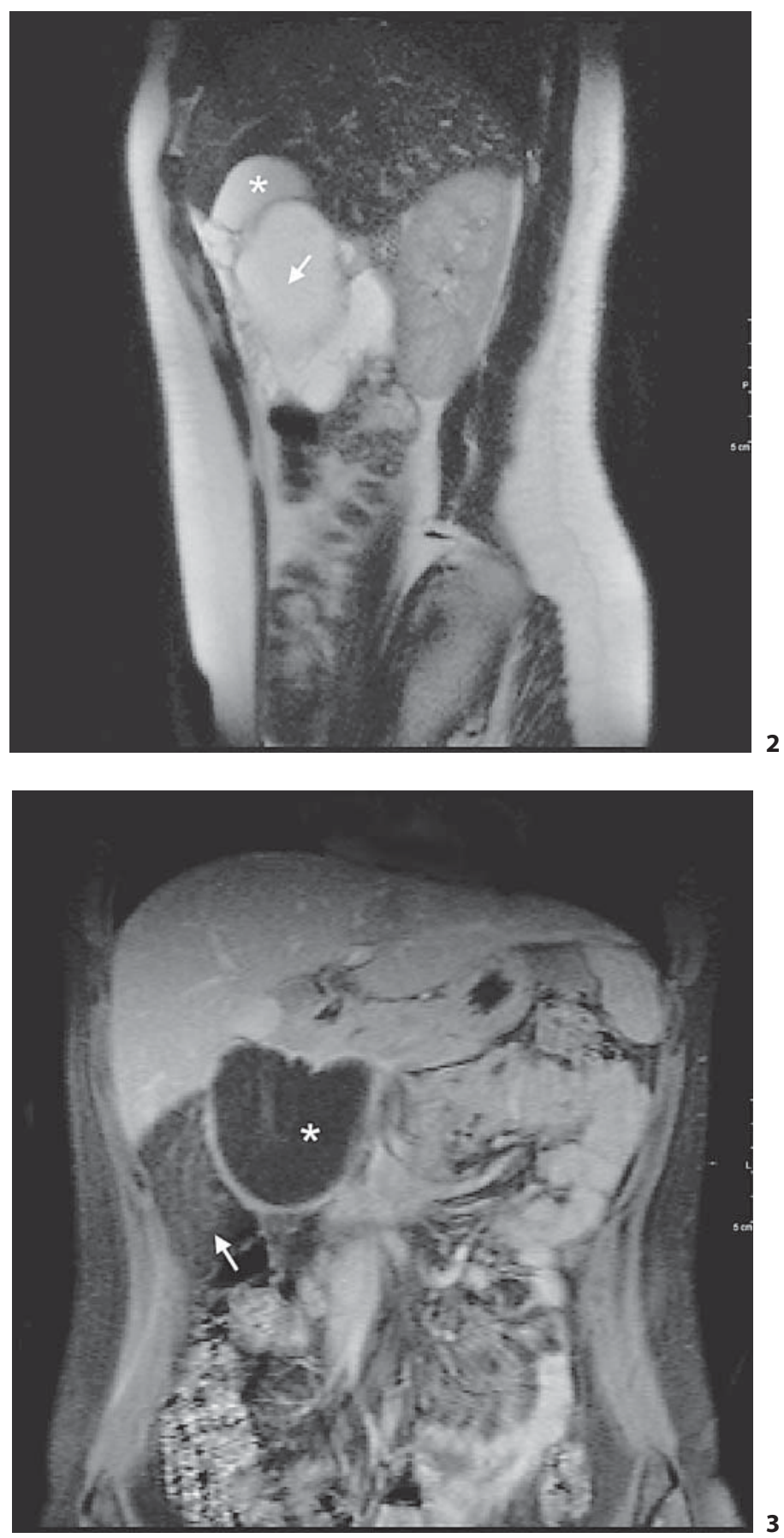

female patients commonly presented the retroperitoneal lymphangioma type. Two cases with lymphangiomas originating from the gallbladder were excluded from this series, because they did not belong to the two types investigated in this study [8]. Sonography and CT were important diagnostic tools for defining location, size, and adjacent organ invasion. 
Acute abdomen is seldom reported in adults, and case reports usually involve the mesenteric type $[9,10]$. Intestinal obstruction was experienced by 2 patients $(25 \%)$ in the mesenteric but by none in the retroperitoneal lymphangioma group ( $p>0.05)$. Acute abdomen is rarely reported in patients with retroperitoneal lymphangiomas, and 2 cases occurred with lymphangiomatosis, as reported in the literature. Yang et al. [11] reported on 1 patient with acute abdomen with a satisfactory outcome. Iwabuchi et al. [12] described a patient with intestinal bleeding having cystic lymphangiomatosis. It is possible that acute abdomen or bleeding occurred frequently in multicentric types [2]. Retroperitoneal cystic lymphangiomas sometimes had a close relationship to visceral organs. Fujishiro et al. [13] reported a patient with a retroperitoneal lymphangioma with dense duodenal adhesion which necessitated pancreaticoduodenectomy. However, the patients in our study showed no retroperitoneal visceral organ invasion.

Results reported in the literature suggest that complete excision is the standard treatment and that resection of the involved organ is necessary. Mesenteric cystic lymphangiomas are usually resected with segments of intestines which can be preserved in patients with retroperitoneal cystic lymphangiomas. Therefore, hospital stay and postoperative course of patients with mesenteric cystic lymphangiomas are longer than in patients with retroperito- neal lymphangiomas. In this study, most patients underwent laparotomy, and the resection margins were negative. With recent advances in laparoscopic surgery, in two reports on cystic lymphangiomas, laparoscopic resection was performed. Laparoscopic surgery was suggested, if indicated $[8,14,15]$.

MRI and CT are common diagnostic modalities, and the typical diagnostic criteria were cystic mass and presence of septa [16]. Two cases were diagnosed by MRI in this study. The presentation on MRI was hypointensity in $\mathrm{T}_{1}$-weighted images and increased intensity in $\mathrm{T}_{2}$-weighted images which represented cystic masses (fig. 1,2). The septal structure was also identified with enhancement after gadolinium injection (fig. 3). However, MRI had a better resolution of tumour and soft tissue [17]. Solomou et al. [18] reported on a splenic lymphangioma detected by MRI, and our such cases were retroperitoneal cystic lymphangiomas.

This report includes a short review of our own experience. Mesenteric and retroperitoneal cystic lymphangiomas had a similar clinical presentation, though the former sometimes presented with intestinal obstruction. The retroperitoneal type frequently occurs in females and at a younger age, whereas the mesenteric type frequently needs intestinal resection. The surgical outcome is generally good with complete resection.

\section{References}

1 Roisman I, Manny J, Fields S, Shiloni E: Intra-abdominal lymphangioma. Br J Surg 1989;76:485-489.

-2 Losanoff JE, Richman BW, El-Sherif A, Rider KD, Jones JW: Mesenteric cystic lymphangioma. J Am Coll Surg 2003;196:598-603.

$\checkmark 3$ Luo CC, Huang CS, Chao HC, Chu SM, Hsueh C: Intra-abdominal cystic lymphangiomas in infancy and childhood. Chang Gung Med J 2004;27:509-514.

4 de Perrot M, Brundler M, Totsch M, Mentha G, Morel P: Mesenteric cysts. Toward less confusion? Dig Surg 2000;17:323-328.

$\checkmark 5$ de Perrot M, Rostan O, Morel P, Le Coultre $\mathrm{C}$ : Abdominal lymphangioma in adults and children. Br J Surg 1998;85:395-397.

-6 Nuzzo G, Lemmo G, Marrocco-Trischitta MM, Boldrini G, Giovannini I: Retroperitoneal cystic lymphangioma. J Surg Oncol 1996;61:234-237.

7 Chau GY, King KL, Su CH, Lui WY: Retroperitoneal cystic lymphangioma in adults. Int Surg 1993;78:243-246.
-8 Yang HR, Jan YY, Huang SF, Yeh TS, Tseng JH, Chen MF: Laparoscopic cholecystectomy for gallbladder lymphangiomas. Surg Endosc 2003;17:1676.

$\checkmark 9$ Matsuba Y, Mizuiri H, Murata T, Niimi K Adult intussusception due to lymphangioma of the colon. J Gastroenterol 2003;38:181185.

10 Chang SC, Tu SH, Shi MY, Huang SH, Chen KM: Mesenteric lymphangioma causing bowel obstruction: report of one case. Acta Paediatr Taiwan 2002;43:43-45.

11 Yang CS, Wu MS, Wang HP, Shun CT, Lin JT: Disseminated cystic lymphangiomatosis presenting with acute abdomen: report of a case and review of the literature. Hepatogastroenterology 1999;46:196-198.

12 Iwabuchi A, Otaka M, Okuyama A, Jin M, Otani S, Itoh S: Disseminated intra-abdominal cystic lymphangiomatosis with severe intestinal bleeding. A case report. J Clin Gastroenterol 1997;25:383-386.

13 Fujishiro M, Kamoshida T, Hotra S, Hirai S, Oka Y, Sato M: Retroperitoneal lymphangioma with a duodenal lesion in an adult. J Gastroenterol 2002;37:381-386.
14 Targarona EM, Moral A, Sabater L, Martinez J, Luque P, Trias M: Laparoscopic resection of a retroperitoneal cystic lymphangioma. Surg Endosc 1994;8:1425-1426.

15 Shimura H, Ueda J, Ogawa Y, Ichimiya H, Tanaka M: Total excision of mesenteric cysts by laparoscopic surgery: report of two cases. Surg Laparosc Endosc 1997;7:173-176.

- 16 Yang DM, Jung DH, Kim H, Kang JH, Kim $\mathrm{SH}$, Kim JH, Hwang HY: Retroperitoneal cystic masses: CT, clinical, and pathologic findings and literature review. Radiographics 2004;24:1353-1365.

17 Feldberg MA, Hendriks AV, Van Leeuwen MS, Witkamp TD, Obertop H: Retroperitoneal cystic lymphangioma section imaging in two cases and review of the literature. Clin Imaging 1990;14:26-30.

18 Solomou EG, Patriarheas GV, Mpadra FA, Karamouzis MV, Dimopoulos I: Asymptomatic adult cystic lymphangioma of the spleen: case report and review of the literature. Magn Reson Imaging 2003;21:81-84. 